\title{
The Role of Hand-Assisted Laparoscopic Surgery in a Right Hemicolectomy for Right-Sided Colon Cancer
}

\author{
Sung Uk Bae, Jin Seok Park ${ }^{1}$, Young Jin $\mathrm{Choi}^{1}$, Min Ku Lee ${ }^{1}$, Byung Sun Cho ${ }^{1}$, Yoon Jung Kang ${ }^{1}$, \\ Joo Seung Park ${ }^{1}$, Chang Nam Kim ${ }^{1}$
}

Division of Colorectal Surgery, Department of Surgery, Colorectal Cancer Clinic, Severance Hospital, Yonsei University College of Medicine, Seoul; ${ }^{1}$ Department of Surgery, Eulji University Hospital, Daejeon, Korea

Purpose: The purpose of this study is to evaluate the perioperative and long-term oncologic outcomes of hand-assisted laparoscopic surgery (HALS) and standard laparoscopic surgery (SLS) and assess the role of HALS in the management of right-sided colon cancer.

Methods: The study group included 53 patients who underwent HALS and 45 patients who underwent SLS for right-sided colon cancer between April 2002 and December 2008.

Results: The patients in each group were similar in age, American Society of Anesthesiologist (ASA) score, body mass index, and history of previous abdominal surgeries. Eight patients in the HALS group and no patient in the SLS group exhibited signs of tumor invasion into adjacent structures. No differences were noted in the time to return of normal bowel function, time to toleration of diet, lengths of hospital stay and narcotic usage, and rate of postoperative complications. The median incision length was longer in the HALS group (HALS: $7.0 \mathrm{~cm}$ vs. SLS: $4.8 \mathrm{~cm}, \mathrm{P}<0.001$ ). The HALS group had a significantly higher pathologic TNM stage and significantly larger tumor size (HALS: $6.0 \mathrm{~cm}$ vs. SLS: $3.3 \mathrm{~cm}, \mathrm{P}<$ 0.001). The 5-year overall, disease-free, and cancer-specific survival rates of the HALS and the SLS groups were $87.3 \%$, $75.2 \%$, and $93.9 \%$ and $86.4 \%, 78.0 \%$, and $90.7 \%$, respectively $(\mathrm{P}=0.826, \mathrm{P}=0.574$, and $\mathrm{P}=0.826)$.

Conclusion: Although patients in the HALS group had more advanced disease and underwent more complex procedures than those in the SLS group, the short-term benefits and the oncologic outcomes between the two groups were comparable. HALS can, therefore, be considered an alternative to SLS for bulky and fixed right-sided colon cancer.

\section{Keywords: Colonic neoplasms; Laparoscopy; Hand-assisted laparoscopy}

\section{INTRODUCTION}

Since the first laparoscopic colectomy was performed by Jacobs in 1990, laparoscopic approaches have become established in the treatment of malignant colorectal diseases [1]. A recent prospec-

Received: June 8,2013 - Accepted: August 21, 2013

Correspondence to: Chang Nam Kim, M.D.

Department of Surgery, Eulji University Hospital, 95 Dunsanseo-ro, Seo-gu, Daejeon 302-799, Korea

Tel: +82-42-259-1335, Fax: +82-42-259-1335

E-mail: kimcn@eulji.ac.kr

(C) 2014 The Korean Society of Coloproctology

This is an open-access article distributed under the terms of the Creative Commons Attribution NonCommercial License (http://creativecommons.org/licenses/by-nc/3.0) which permits unrestricted noncommercial use, distribution, and reproduction in any medium, provided the original work is properly cited. tive, multi-institutional, randomized study showed that the laparoscopic approach was an acceptable alternative to open surgery for colon cancer [2-4]. Lacy et al. [5] showed that standard laparoscopic surgery (SLS) was preferred over open surgery for patients with colon cancer because it reduced perioperative morbidity, shortened the length of stay in the hospital, and prolonged cancerrelated survival. However, these studies were conducted with groups of patients that were heterogeneous in terms of tumor location. Unlike a laparoscopic cholecystectomy, which has rapidly become the standard of care, laparoscopic colorectal surgery has not become established as the procedure of choice for many reasons, including a steep learning curve for surgeons, uncertainty about the procedure's effectiveness for malignancy, the need to operate in multiple quadrants of the abdomen, a long operating time, and a lack of tactile feedback. 
Hand-assisted laparoscopic surgery (HALS) was developed as an alternative to SLS for the treatment of colorectal diseases. Studies comparing the results of HALS and SLS, including several prospective randomized trials, have found that HALS maintains the beneficial features of SLS with no differences in terms of pain, duration of ileus, time until patient's return to normal activity, or length of hospital stay [6-8]. Marcello et al. [8] showed that HALS resulted in significantly shorter operation times and reduced the need for conversion to open surgery in patients undergoing a leftsided colectomy and a total abdominal colectomy. Our study aimed to compare the efficacies of HALS and SLS in an elective laparoscopic right hemicolectomy (RHC) for colon cancer in terms of perioperative outcomes and long-term oncologic outcomes and to assess the role of HALS in the management of rightsided colon cancer.

\section{METHODS}

\section{Patients}

From April 2002 to December 2008, a total of 151 patients underwent a RHC for right-sided colon cancer at Eulji University Hospital. After the exclusion criteria had been applied, the study group consisted of 53 patients who underwent HALS and 45 patients who underwent SLS. The exclusion criteria were active inflammatory disease (intra-abdominal abscess or sepsis), bowel perforation, distant metastasis, and synchronous or metachronous malignancy. The patients' demographics and the perioperative and oncologic outcomes were obtained from a prospectivelycollected Eulji University Colorectal Cancer Database. Prospectively-collected data included perioperative features, including operative time, incision length and conversion rate; the patient's immediate clinical response, including recovery of bowel sounds, refeeding time, postoperative pain, hospital stay, and postoperative complications; and oncological characteristics, including features of the specimen and the number of lymph nodes harvested. A conversion of laparoscopic surgery was defined as an interruption of the minimally-invasive approach, followed by the need for a laparotomy at any time to complete the surgical procedure. Recurrence was defined as the presence of a radiologically- and/or histologically-confirmed tumor, and the location of recurrence was defined as the first site of recurrence after a complete resection. Unless contraindicated, postoperative adjuvant chemotherapy using standard schedules and doses was routinely given to all patients with stages II and III tumors.

\section{Operative technique}

For SLS, under general anesthesia, the patient was placed in a modified lithotomy position, and a urinary catheter was placed in the bladder. The first $10-\mathrm{mm}$ port was placed via a cut-down procedure and was positioned in the patient infraumbilically, allowing for the establishment of pneumoperitoneum (10-12 mmHg). After laparoscopic exploration of the abdominal cavity, four addi- tional 5-mm ports were placed under direct visualization: two 5 -mm ports, one each in the upper and the lower left quadrants, for the surgeon and two 5-mm ports, one each in the upper and the lower right quadrants, for the surgical assistant (Fig. 1A). Out of 45 patients who underwent SLS, 41 underwent a RHC using a retroperitoneal approach. Instead of placing two 5-mm ports in the right lower quadrant, we made a $10-\mathrm{mm}$ muscle-splitting incision, two-finger breadth, above the iliac crest, and we created a tunnel down to the retroperitoneal space (Fig. 1B). A retroperitoneal cavity was created using an OMS-PDBS2 distension balloon (Covidien, Norwalk, CT, USA). After the distension balloon had been removed, a blunt-tipped balloon trocar (OMS-T10BT, Covidien) was inserted for retroperitoneoscopy, and the retroperitoneum was insufflated with $\mathrm{CO}_{2}$ up to a maximum cavity pressure of $10 \mathrm{mmHg}$. After the pneumoretroperitoneum had been established, two secondary 5-mm trocars were inserted, one each in the upper and the lower portions of the optic trocar, and the retroperitoneal approach was initiated. A dissection was performed at the level of the duodenum to the right common iliac artery with identification of the ureter, taking care to avoid injury. The lateral-running ileocolic/right colic arteries of the branches of the superior mesenteric artery were identified, exposed, and marked using a clip applier, and the retroperitoneal approach was completed. In the transperitoneal approach, two 5-mm ports were used for a surgical assistant. The patient was placed in a Trendelenburg position with a tilted left-side-down position. The right mesocolon was divided in a plane that was to the right of the superior mesenteric axis, and the ileocolic vessels, right colic vessels, and right branch of the middle colic artery were divided at the level of the origin based on the concept of central vascular ligation. An avascular surgical plane composed of Toldt's and prerenal fascia was exposed to uncover the head of the pancreas, a second portion of the duodenum, right gonadal vessels, and the ure-
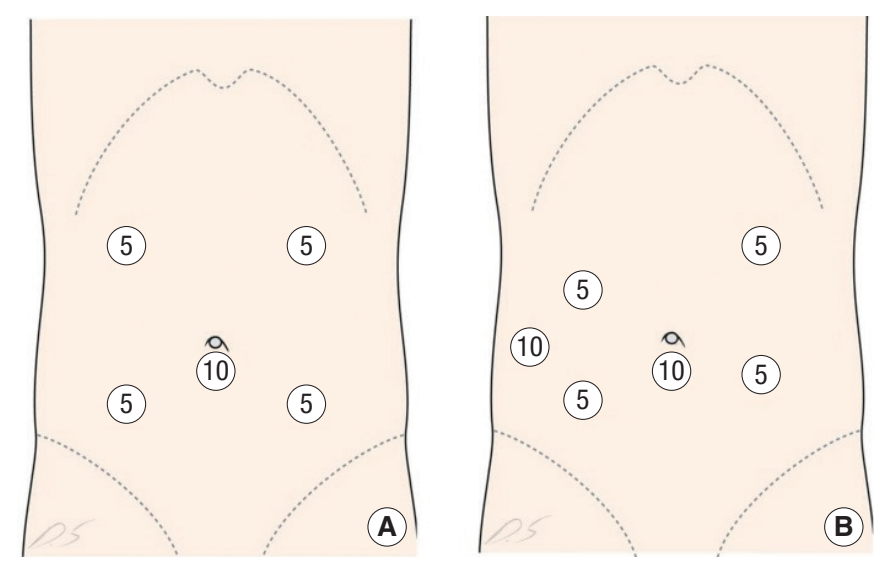

Fig. 1. Port sites in the standard laparoscopic surgery (SLS) group (dimensions in millimeters). (A) Port sites in the SLS group for the transabdominal approach. (B) Port sites in the SLS group for the transabdominal and retroperitoneal approach. 
ter. The integrity of the mesocolon was strictly preserved, similar to a total mesorectal excision (TME) for rectal cancer based on the concept of a complete mesocolic excision. After detachment of the lateral peritoneum of the ascending colon and the attachment of the hepatic flexure, the transverse mesocolon was divided toward the site where the transverse colon divided. After proper mobilization, a periumbilical midline incision, $4.5 \mathrm{~cm}$ in length, was made around the left side of the umbilicus. The colon from the terminal ileum to the midtransverse colon was exteriorized through the incision, and ileocolic anastomosis was performed extracorporeally by either hand sewing or completion with two linear staples. The anastomosis was placed back into the peritoneal cavity, and saline irrigation was performed. The periumbilical incision was closed in three layers, the peritoneum, fascia, and skin.

In HALS, the first port was placed via a cut-down procedure and was positioned infraumbilically and a LAP DISC (Ethicon Endosurgery Inc., Cincinnati, OH, USA) was placed through a 7 to 7.5 $\mathrm{cm}$ midline incision between the xiphoid process and the umbilicus. After pneumoperitoneum (10-12 $\mathrm{mmHg}$ ) was established (Fig. 2), laparoscopic exploration of the abdominal cavity was performed. Three additional 5-mm ports were placed under direct visualization, one in the left lower quadrant for the surgeon and two, one each in the right upper and lower quadrants for a surgical assistant. The bowel was properly mobilized and then exteriorized through the HALS incision, and the bowel was divided and reanastomosed by using conventional open techniques.

\section{Statistical analyses}

Clinical characteristics and variables were compared between the HALS and the SLS groups by using an independent-sample t-test for continuous data or a chi-square test for categorical data. Overall survival was defined as time from surgery to death from any cause, and for estimates of disease-free survival, patients who died without having disease recurrence were censored at the time of death. Analysis of survival was performed using the Kaplan-Meier

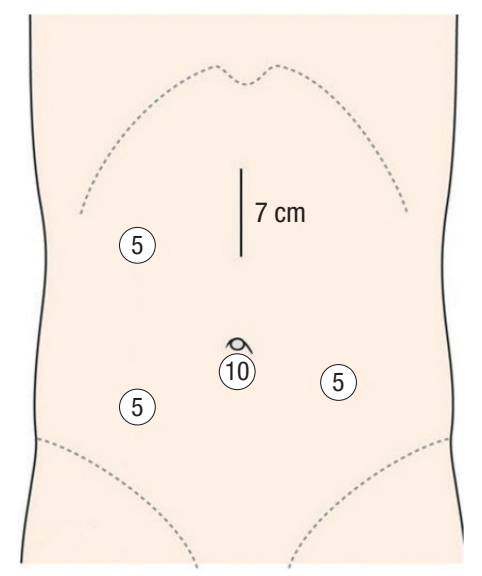

Fig. 2. Port sites in the hand-assisted laparoscopic surgery group (dimensions in millimeters). method, and differences between the curves were assessed by using the log-rank test. P-values of less than 0.05 were considered to indicate statistical significance. Analyses were performed using SPSS ver. 12.0 (SPSS Inc., Chicago, IL, USA).

\section{RESULTS}

\section{Patient characteristics}

The median lengths of follow-up were 43 months (2-84 months) for the whole study population, 42 months (2-77 months) for the HALS group, and 48 months (12-85 months) for the SLS group (P $=0.140)$. Demographic characteristics were similar between the two groups with respect to age, preoperative carcinoembryonic antigen, American Society of Anesthesiologists grade, body mass index, and the presence of previous abdominal surgery (Table 1). There was no intraoperative evidence of tumor invasion to adjacent organs in the SLS group whereas eight patients in the HALS group exhibited signs of tumor invasion into adjacent structures including the peritoneum (6 cases), small bowel ( 1 case), and duodenum (1 case), and a combined resection of other organs was performed in these cases.

\section{Clinical short-term outcomes}

No significant differences in the median operation times were noted between the HALS and the SLS groups (318 minutes vs. 300 minutes, $\mathrm{P}=0.330$ ), and there were two conversions to open surgery in each group. No apparent differences in the time taken to return of normal bowel function, tolerance of diet, length of hospital stay, or narcotic usage were noted between the two groups, but the SLS group had a significantly smaller incision for speci-

Table 1. Patient baseline clinical characteristics

\begin{tabular}{lccc}
\hline Characteristic & HALS $(\mathrm{n}=53)$ & SLS $(\mathrm{n}=45)$ & P-value \\
\hline Age (yr) & $68(30-85)$ & $63(36-90)$ & 0.354 \\
Sex & & & 0.030 \\
$\quad$ Male & $34(64.2)$ & $19(42.2)$ & \\
$\quad$ Female & $19(35.8)$ & $26(57.8)$ & \\
Preoperative CEA (ng/mL) & $2.5(0.1-64.3)$ & $1.94(0.1-10.4)$ & 0.093 \\
ASA group & & & 0.713 \\
I & $18(34.0)$ & $16(35.6)$ & \\
॥ & $23(43.4)$ & $23(51.1)$ & \\
III & $10(18.9)$ & $6(13.3)$ & \\
$\quad$ IV & $2(3.8)$ & $0(0)$ & \\
Body mass index (kg/m²) & $22.9(16.2-32.9)$ & $23.6(18.7-32.3)$ & 0.404 \\
Previous abdominal & $17(32.1)$ & $14(31.1)$ & 0.816 \\
$\quad$ surgeries & & & \\
Invasion to adjacent organs & 8 & 0 & $<0.001$ \\
\hline
\end{tabular}

Values are presented as median (range) or number (\%).

HALS, hand-assisted laparoscopic surgery; SLS, standard laparoscopic surgery; CEA, carcinoembryonic antigen; ASA, American Society of Anesthesiologists. 
men extraction (HALS: $7.0 \mathrm{~cm}$ vs. SLS: $4.8 \mathrm{~cm}, \mathrm{P}<0.001$ ). No significant differences in the rates of postoperative complications were noted between the groups (17.0\% in the HALS group and $12.9 \%$ in the SLS group, $\mathrm{P}=0.617$ ). Nine patients in the HALS group had complications: two patients underwent reoperation due to anastomosis dehiscence whereas the other seven patients, wound infection ( 2 cases), a ventral hernia (1 case), or ileus (4 cases), were treated conservatively. Six patients in the SLS group had complications: one patient underwent reoperation due to anastomosis dehiscence while five patients, wound infection (1 case), acute cholecystitis (1 case), or ileus (3 cases), were treated conservatively (Table 2). No mortalities occurred within 30 days.

\section{Postoperative pathologic results}

The HALS group had a significantly higher pathologic TNM stage (HALS: stage $0, \mathrm{n}=2 ; \mathrm{I}, \mathrm{n}=5 ; \mathrm{II}, \mathrm{n}=28 ; \mathrm{III}, \mathrm{n}=18$ vs. SLS: stage $0, \mathrm{n}=$ 11; I, $\mathrm{n}=15 ;$ II, $\mathrm{n}=9$; III, $\mathrm{n}=10 ; \mathrm{P}<0.001$ ) and significantly larger median tumor size (HALS: $6.0 \mathrm{~cm}$ vs. SLS: $3.3 \mathrm{~cm}, \mathrm{P}<0.001$ ) than the SLS group (Table 3 ). The median numbers of harvested lymph nodes were similar in the two groups (HALS: 39 vs. SLS: 36, P = $0.534)$.

\section{Oncologic outcomes}

Overall, 13 patients had recurrence of the tumor (7 in the HALS group and 6 in the SLS group), and 11 patients died (6 in the HALS group and 5 in the SLS group) (Table 4). The 5 -year overall

Table 2. Perioperative outcomes

\begin{tabular}{lccc}
\hline Variable & HALS $(\mathrm{n}=53)$ & SLS $(\mathrm{n}=45)$ & P-value \\
\hline Operation time (min) & $318(180-535)$ & $300(200-550)$ & 0.330 \\
First flatus POD & $3(1-6)$ & $3(1-5)$ & 0.724 \\
Time-to-liquid diet (day) & $4(3-11)$ & $4(3-18)$ & 0.932 \\
$\begin{array}{l}\text { Duration of narcotic } \\
\text { analgesia (day) }\end{array}$ & $3(0-10)$ & $3(0-10)$ & 0.577 \\
Hospital stay (day) & $11(8-56)$ & $10(8-40)$ & 0.166 \\
Incision length (cm) & $7.0(6-12)$ & $4.8(3-7)$ & $<0.001$ \\
Conversion & $1(1.9)$ & $1(2.2)$ & 0.907 \\
Morbidity within 30 days & & & \\
$\quad$ after surgery & & & \\
Overall & $9(17.0)$ & $6(12.9)$ & 0.617 \\
$\quad$ lleus & $4(7.5)$ & $3(6.7)$ & \\
$\quad$ Anastomotic leakage & $2(3.8)$ & $1(2.2)$ & \\
$\quad$ Acute cholecystitis & $0(0)$ & $1(2.2)$ & \\
$\quad$ Wound infection & $2(3.8)$ & $1(2.2)$ & \\
$\quad$ Vental hernia & $1(2.2)$ & $0(0)$ & \\
Mortality within 30 days after & $0(0)$ & $0(0)$ & \\
surgery & & & \\
\hline
\end{tabular}

Values are presented as number (\%) or median (range).

HALS, hand-assisted laparoscopic surgery; SLS, standard laparoscopic surgery; POD, postoperative day. survival rates of the HALS and the SLS groups were $87.3 \%$ and $86.4 \%$, respectively $(\mathrm{P}=0.826)$, and the 5 -year disease-free survival rates were $75.2 \%$ and $78.0 \%(\mathrm{P}=0.574)$ (Fig. 3). The 5-year cancerspecific survival rates of the HALS and the SLS groups were 93.9\% and $90.7 \%$, respectively $(\mathrm{P}=0.826)$, and the overall rates of recurrence did not differ significantly between the HALS group and the SLS group (13.2\% vs. $13.3 \%, \mathrm{P}=0.985)$.

\section{DISCUSSION}

SLS has been shown to have multiple short-term patient benefits, including decreased postoperative pain, shorter hospital stay, earlier return of gastrointestinal function, faster return to normal activity, smaller incision, and fewer postoperative pulmonary and wound complications. On the other hand, the limitations of a purely laparoscopic approach include a relative lack of tactile feedback and the absence of depth perception; other limitations include the fact that SLS is a demanding technique with a steep learning curve and a longer operative time than open surgery, and

Table 3. Pathological characteristics of resected tumors

\begin{tabular}{lccc}
\hline Variable & HALS $(\mathrm{n}=53)$ & SLS $(\mathrm{n}=45)$ & P-value \\
\hline Histology & & & 0.015 \\
Well-differentiated & $5(9.4)$ & $15(33.3)$ & \\
Moderately differentiated & $40(75.4)$ & $27(60.0)$ & \\
Poorly differentiated & $6(11.3)$ & $3(6.7)$ & \\
Mucinous & $2(3.9)$ & $0(0)$ & \\
TNM Stage & & & $<0.001$ \\
O & $2(3.8)$ & $11(24.4)$ & \\
I & $5(9.4)$ & $15(33.3)$ & \\
॥I & $28(52.8)$ & $9(20.0)$ & \\
III & $18(34.0)$ & $10(22.2)$ & \\
Tumor size (cm) & $6.0(1.3-12.5)$ & $3.3(0.5-9.0)$ & $<0.001$ \\
Retrieved LNs & $39(5-111)$ & $36(21-114)$ & 0.534 \\
LVI (+) & $34(64.2)$ & $21(46.7)$ & 0.082 \\
\hline
\end{tabular}

Values are presented as number (\%) or median (range).

HALS, hand-assisted laparoscopic surgery; SLS, standard laparoscopic surgery; LNs, lymph nodes; LVI, lymphovascular invasion.

Table 4. Five-year oncologic outcomes for standard and hand-assisted laparoscopic RHCs

\begin{tabular}{lccc}
\hline Variable & HALS $(\mathrm{n}=53)$ & $\mathrm{SLS}(\mathrm{n}=45)$ & P-value \\
\hline Overall survival & 87.3 & 86.4 & 0.826 \\
Disease-free survival & 75.2 & 78.0 & 0.574 \\
Cancer-specific survival & 93.9 & 90.7 & 0.966 \\
Overall rates of recurrence & $7(13.2)$ & $6(13.3)$ & 0.985
\end{tabular}

Values are presented as percentage or number (\%).

$\mathrm{RHC}$, right hemicolectomy; HALS, hand-assisted laparoscopic surgery; SLS, standard laparoscopic surgery. 

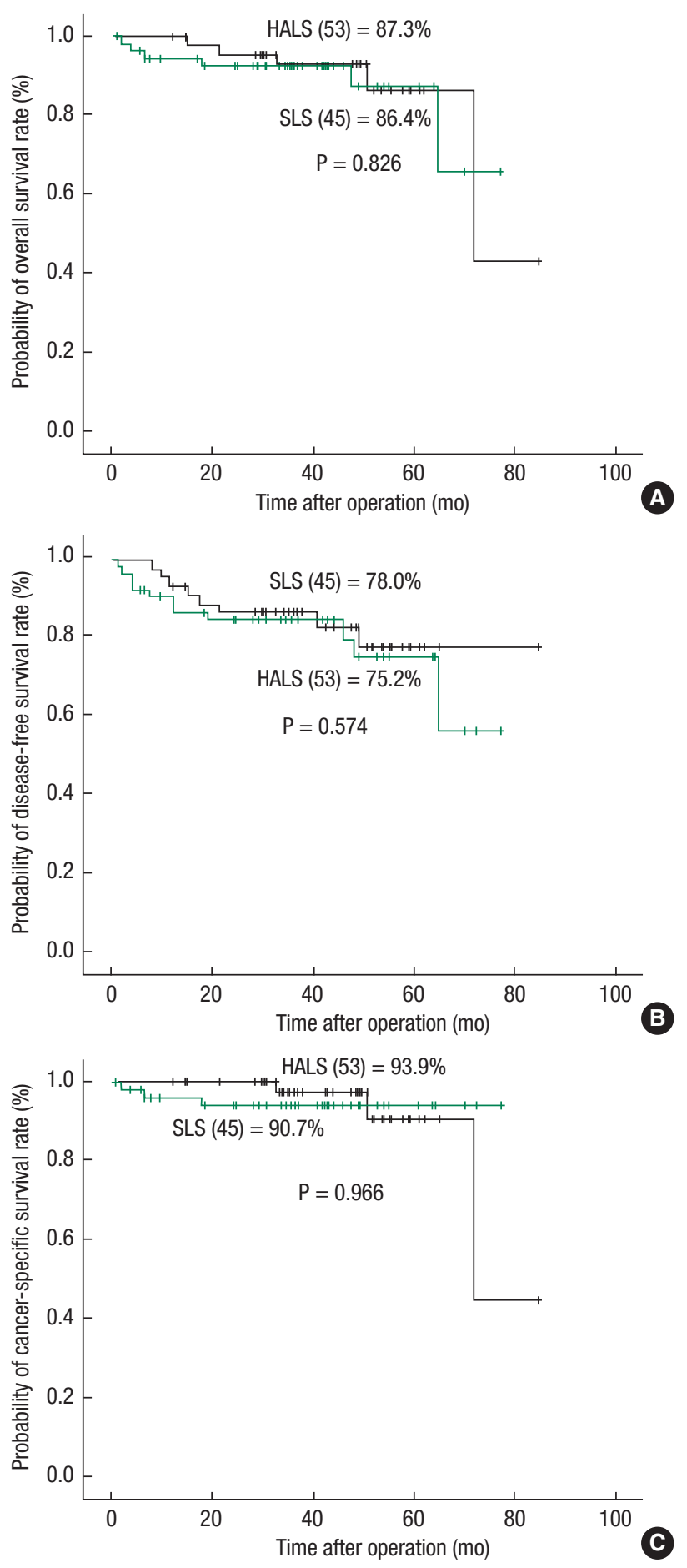

Fig. 3. Survival after surgery. (A) Overall survival rates, (B) diseasefree survival rates, and $(\mathrm{C})$ cancer-specific survival rates between the SLS and the HALS groups. HALS, hand-assisted laparoscopic surgery; SLS, standard laparoscopic surgery.

the absence of gentle and safe laparoscopic retracting devices.

HALS allows the surgeon to place a nondominant hand within the abdominal cavity to assist in the use of laparoscopic instrumentation. A hand-assisted laparoscopic colectomy has been described as a hybrid procedure [9] and was the first report of the HALS concept [10]. Several advantages of the hand-assisted approach were emphasized: the inserted hand can provide blunt dissection, tactile feedback from the hand helps to identify lesions that are not clearly visible, and better control of bleeding and better organ retraction are possible. HALS, therefore, represents a useful alternative to SLS $[6,11]$ and may be considered as a new method for more complex procedures or more difficult pathologies [7, 12, 13]. However, the best use for HALS is laparoscopy-assisted surgery in which an additional incision is required for extracting the specimen. Therefore, HALS seems to be especially suited for colorectal surgery $[6,7,11]$.

Although HALS has been considered a means to help nonskilled surgeons through the long learning curve associated with performing a laparoscopic colectomy $[11,14,15]$, both HALS and SLS have made unique individual contributions to surgery. In this study, we view HALS as another tool in the surgeon's armamentarium that can be used in selected patients.

The location of the port placement is important because it can determine the range of blunt hand dissection whereas inappropriate port placement for the hand device may compromise the view of the camera. A trial [8] of HALS and SLS for a laparoscopic RHC showed that patients who were this or had a short stature were better suited for SLS, as port placement for the hand device through the midline or Pfannenstiel incision might compromise the view of the camera. On the other hand, in the study of Schadde et al. [16], the handport device was placed in the midline, and a 10-mm trocar was placed in the right upper quadrant for the camera, and Chung et al. [17] suggested handport device placement through a lower abdominal midline incision. Ballantyne and Leahy [18] preferred to place the handport device through an upper abdominal midline incision near the planned anastomosis, similar to the position of our handport device. The position of the port placement was determined by the location of the lesion, the location of anastomosis, and the side of the surgeon's dominant hand. If the surgeon can use both his or her nondominant hand and dominant hand in the abdominal cavity for HALS, in our opinion, this position of the handport placement is appropriate for a hand-assisted laparoscopic RHC.

In the current study, the surgeon inserted the handport device at the beginning of the procedure and then used it during the remainder of the operation. On the other hand, in the report of Chew and Adams [19], the surgeon's left hand was placed through the Pfannenstiel incision, and the ascending colon, hepatic flexure, and proximal transverse colon were mobilized bluntly by using the left hand, which was then withdrawn from the handport device and replaced by diathermy scissors at the dissection of the lateral peritoneal attachments.

Several technical aspects are related to HALS. Lee et al. [20] showed that a moist laparotomy pad could be inserted through 
the handport device and was used to gently pack the small intestine away from the pelvis. Nakajima et al. [21] also described how surgical towels could be used to move the small intestine away from the operative site. Most handport devices could function as a wound protector, which should theoretically protect the HAL wound from tumor implantation. Recently, a modified laparoscopic technique was developed and is referred to as the HALS Finger-Mounted Instrument (Ethicon Endo-surgery Inc., Cincinnati, $\mathrm{OH}, \mathrm{USA}$ ), in which miniature forceps, graspers, scissors, dissectors, probes and retractors mounted on the surgeon's fingers can perform delicate surgical tasks.

In this study, 41 of 45 patients who underwent SLS underwent a RHC using a retroperitoneal approach. The retroperitoneal approach for a RHC is not popular and needs an additional $10-\mathrm{mm}$ port. However, that approach, which takes about 15 minutes, is similar to the lateral or inferior approach in the sense that the dissection around the main vessels is performed after the dissection of retroperitoneal structures, such as the ureter, gonadal vessels, and the duodenum. The following are some advantages compared to the transabdominal approach:

- Much of the retroperitoneal cavity can be created by only air insertion thorough the distension balloon.

- An identification and dissection of the ureter and the gonadal vessels can be easily performed.

- The surgical field is not disturbed by the small bowel.

- The range of the dissection after the medial-to-lateral transabdominal approach can be decreased.

In our institute, this approach is commonly performed in a RHC except for (1) tumors larger than $6 \mathrm{~cm}$ and (2) tumors locally invading adjacent organs.

A previous prospective, randomized, multicenter study concluded that HALS had similar short-term clinical outcomes to SLS and resulted in significantly shorter operation times than SLS for patients who underwent a left-sided colectomy and a total abdominal colectomy [8]. In the present study, no significant difference in the median operation times were noted between the HALS and the SLS groups ( 318 minutes vs. 300 minutes, $\mathrm{P}=0.330$ ). The selection of patients for one approach versus the other was subject to significant selection biases, such as patient characteristics, disease and operative factors, and the surgeon's experience. The HALS resection group had a significantly higher pathologic TNM stage, which resulted in a significantly larger tumor size, than the SLS resection group. The patients in the HALS group were more likely than those in the SLS group to undergo concurrent resection of adjacent organs because of tumor invasion, reflecting the increased complexity of HALS procedures. Therefore, considering that more advanced tumors were prevalent in the HALS group compared with SLS group, the similar perioperative outcomes and long-term oncologic outcomes between the two groups of patients suggest that HALS is beneficial in selected patients.

Consistent with other studies showing that HALS allows surgeons to perform more extensive and complex procedures, such as a lapa- roscopic total colectomy or proctocolectomy [22-24], we found that use of HALS in a RHC for right-sided colon cancer allowed us to expand our minimally-invasive practice to patients who might not otherwise have been considered candidates for SLS while still retaining the perioperative patient-related benefits of minimally invasive surgery. Indications of HALS for colorectal diseases in our clinic are (1) cases in which tactile feedback is necessary, like hepatic metastasis and uncertain state of intestine, (2) cases in which force is necessary, like firm adhesion, invasion to adjacent organs, (3) difficult surgeries, like adhesive ileus and obstructing colon cancer, (4) time-consuming surgeries, like a total colectomy and a total proctocolectomy, (5) tumors larger than $6 \mathrm{~cm}$, (6) surgeon's preference, HALS also being used as a routine procedure when the surgeon prefers that approach, and (7) patient with surgical and anesthetic high-risk to shorten the operation time.

The limitations of this study include its retrospective nature, the small size of the study, and the difference of pathologic TNM stage between the two groups. Therefore, a large, randomized, prospective study comparing short- and long-term outcomes between HALS and SLS for right-sided colon cancer is needed.

In conclusion, although patients in the HALS group had more advanced disease and underwent more complex procedures than those in the SLS group, the short-term benefits and the oncologic outcomes between the two groups were comparable. HALS can, therefore, be considered an alternative to SLS for bulky and fixed right-sided colon cancer.

\section{CONFLICT OF INTEREST}

No potential conflict of interest relevant to this article was reported.

\section{REFERENCES}

1. Jacobs M, Verdeja JC, Goldstein HS. Minimally invasive colon resection (laparoscopic colectomy). Surg Laparosc Endosc 1991;1: 144-50.

2. Laparoscopically assisted colectomy is as safe and effective as open colectomy in people with colon cancer Abstracted from: Nelson H, Sargent D, Wieand HS, et al; for the Clinical Outcomes of Surgical Therapy Study Group. A comparison of laparoscopically assisted and open colectomy for colon cancer. N Engl J Med 2004;350:2050-2059. Cancer Treat Rev 2004;30:707-9.

3. Jayne DG, Guillou PJ, Thorpe H, Quirke P, Copeland J, Smith $\mathrm{AM}$, et al. Randomized trial of laparoscopic-assisted resection of colorectal carcinoma: 3-year results of the UK MRC CLASICC Trial Group. J Clin Oncol 2007;25:3061-8.

4. COLOR Study Group. COLOR: a randomized clinical trial comparing laparoscopic and open resection for colon cancer. Dig Surg 2000;17:617-22.

5. Lacy AM, Garcia-Valdecasas JC, Delgado S, Castells A, Taura P, Pique JM, et al. Laparoscopy-assisted colectomy versus open col- 
ectomy for treatment of non-metastatic colon cancer: a randomised trial. Lancet 2002;359:2224-9.

6. Targarona EM, Gracia E, Garriga J, Martinez-Bru C, Cortes M, Boluda R, et al. Prospective randomized trial comparing conventional laparoscopic colectomy with hand-assisted laparoscopic colectomy: applicability, immediate clinical outcome, inflammatory response, and cost. Surg Endosc 2002;16:234-9.

7. Hand-assisted laparoscopic surgery vs standard laparoscopic surgery for colorectal disease: a prospective randomized trial. HALS Study Group. Surg Endosc 2000;14:896-901.

8. Marcello PW, Fleshman JW, Milsom JW, Read TE, Arnell TD, Birnbaum EH, et al. Hand-assisted laparoscopic vs. laparoscopic colorectal surgery: a multicenter, prospective, randomized trial. Dis Colon Rectum 2008;51:818-26.

9. Romanelli JR, Kelly JJ, Litwin DE. Hand-assisted laparoscopic surgery in the United States: an overview. Semin Laparosc Surg 2001;8:96-103.

10. $\mathrm{Ou} \mathrm{H}$. Laparoscopic-assisted mini laparatomy with colectomy. Dis Colon Rectum 1995;38:324-6.

11. Litwin DE, Darzi A, Jakimowicz J, Kelly JJ, Arvidsson D, Hansen P, et al. Hand-assisted laparoscopic surgery (HALS) with the HandPort system: initial experience with 68 patients. Ann Surg 2000; 231:715-23.

12. Hassan I, You YN, Cima RR, Larson DW, Dozois EJ, Barnes SA, et al. Hand-assisted versus laparoscopic-assisted colorectal surgery: practice patterns and clinical outcomes in a minimally-invasive colorectal practice. Surg Endosc 2008;22:739-43.

13. Nakajima K, Nezu R, Hirota M, Nishida T. The role of hand-assisted laparoscopic surgery in subtotal and total colectomy for Crohn's colitis. Surg Endosc 2010;24:2713-7.

14. Chang YJ, Marcello PW, Rusin LC, Roberts PL, Schoetz DJ. Hand-assisted laparoscopic sigmoid colectomy: helping hand or hindrance? Surg Endosc 2005;19:656-61.
15. Takakura Y, Okajima M, Yoshimitsu M, Hinoi T, Ikeda S, Ohdan $\mathrm{H}$. Hybrid hand-assisted colectomy for transverse colon cancer: a useful technique for non-expert laparoscopic surgeons. World J Surg 2009;33:2683-7.

16. Schadde E, Smith D, Alkoraishi AS, Begos DG. Hand-assisted laparoscopic colorectal surgery (HALS) at a community hospital: a prospective analysis of 104 consecutive cases. Surg Endosc 2006;20:1077-82.

17. Chung CC, Ng DC, Tsang WW, Tang WL, Yau KK, Cheung HY, et al. Hand-assisted laparoscopic versus open right colectomy: a randomized controlled trial. Ann Surg 2007;246:728-33.

18. Ballantyne GH, Leahy PF. Hand-assisted laparoscopic colectomy: evolution to a clinically useful technique. Dis Colon Rectum 2004;47:753-65.

19. Chew SS, Adams WJ. Laparoscopic hand-assisted extended right hemicolectomy for cancer management. Surg Endosc 2007;21: 1654-6.

20. Lee SW, Sonoda T, Milsom JW. Expediting of laparoscopic rectal dissection using a hand-access device. Dis Colon Rectum 2007; 50:927-9.

21. Nakajima K, Milsom JW, Margolin DA, Szilagy EJ. Use of the surgical towel in colorectal hand-assisted laparoscopic surgery (HALS). Surg Endosc 2004;18:552-3.

22. Agachan F, Joo JS, Sher M, Weiss EG, Nogueras JJ, Wexner SD. Laparoscopic colorectal surgery. Do we get faster? Surg Endosc 1997;11:331-5.

23. Nakajima K, Lee SW, Cocilovo C, Foglia C, Kim K, Sonoda T, et al. Hand-assisted laparoscopic colorectal surgery using GelPort. Surg Endosc 2004;18:102-5.

24. Watanabe K, Funayama Y, Fukushima K, Shibata C, Takahashi K, Sasaki I. Hand-assisted laparoscopic vs. open subtotal colectomy for severe ulcerative colitis. Dis Colon Rectum 2009;52:640-5. 